is recruited to DNA double strand breaks and is required for ATM activation. - In response to doxorubicin, ATM activation is dependent on ING3 but not on TIP60, whose recruitment to DNA breaks also depends on ING3. - These events lead to ATM-mediated phosphorylation of NBS1 and of major mediators of the DNA damage response. - Upon genotoxic stress, DNA repair by Non Homologous End Joining (NHEJ) or Homologous Recombination (HR) were impaired in absence of ING3. - Immunoglobulin Class Switch Recombination (CSR), a physiological mechanism requiring NHEJ repair, was impaired in the absence of ING3. Conclusions: Since deregulation of DNA double strand break repair is associated with genomic instability, we propose a novel function of ING3 as a caretaker tumor suppressor involved in the DNA damage signaling and repair.

A.M. was a recipient of a doctoral fellowship from $\mathrm{La}$ Ligue Contre le Cancer and Region Bretagne. R.P. \& M.G. were recipients of from Ulysses "The France - Ireland Exchange Scheme". N. Bigot was a recipient of ANR program (SAFE 2012) (ANR-11-RPIB-0012). C.

References: Cell Death Differ. 2019 Feb 25. doi: 10.1038/s41418-019-0305-x. [Epub ahead of print]

doi: http://dx.doi.org/10.7124/bc.0009BE

\section{Computer modeling of phase separation at PML nuclear bodies}

Richard Henze, Marvin Wenzel, Peter Dittrich \& $\underline{\text { Peter Hemmerich }}$

Leibniz Institute on Aging

peter.hemmerich@leibniz-fli.de

The biophysical mechanism of liquid-liquid phase separation (LLPS) has emerged as an attractive idea to explain the formation and function of membrane-less organelles, such as nuclear bodies. Our aim is to simulate the genesis of promyelocytic leukemia nuclear bodies (PML-NBs) at the molecular level using computer models in order to gain insight on LLPS within PML-NBs. To this end, we have generated a computer model of PML-NB assembly which uncovers molecular details of their genesis and a spatial map of its LLPSdriving elements. Model predictions can be exploited to test new hypotheses of PML-NB structure in the wet lab in an iterative process. The established computer model may be expanded to other membrane-less organelles to reveal their structural details.

doi: http://dx.doi.org/10.7124/bc.0009BF

\section{Interplay of DNA replication, repair and chromatin}

M. Cristina Cardoso

Cell Biology and Epigenetics, Dept. Biology, TU

Darmstadt, Germany

cardoso@bio.tu-darmstadt.de

Half a century ago the elucidation of the DNA double helix structure was quickly followed by the visualization of replicons in DNA fibers. To connect 1D DNA replication/repair information with whole cell 3D data in mammalian cells, we combined super resolution microscopy and time-lapse analysis of S-phase dynamics with genome size and DNA replication fiber analysis. We found that the subnuclear replication structures can be optically resolved down to single replicons during all S-phase stages. This sets aside the conventional inter- 
pretation of nuclear replication foci as replication factories, i.e., the complex entities that process multiple clustered replicons. Further, our data suggest that S-phase dynamics is primarily dictated by chromatin folding and synthetic replisome complexes assemble on template DNA. Accordingly, individual replicons within the chromatin context represent the elementary units of 3D genome duplication and organization. A similar analysis of DNA damage response after ionizing radiation labeled by phosphorylated H2AX, a widely used DNA double strand break signaling marker, yielded (nano)foci of the same order of magnitude. These data raise the hypothesis that both repair and replication structures reflect the same elementary unit of chromatin organization. Finally, we manipulated nuclear chromatin (com)position and found that it affected DNA replication dynamics and it ultimately lead to changes in chromatin composition.

References: Löb, D., Lengert, N., Chagin, V. O, Reinhart, M., Casas-Delucchi, C. S., Cardoso, M. C. and Drossel, B. (2016). 3D replicon distributions arise from stochastic initiation and domino-like DNA replication progression. Nat. Commun. 7: 11207. Chagin, V. O., Casas-Delucchi, C. S., Reinhart, M., Schermelleh, L., Markaki, Y., Maiser, A., Bolius, J. J., Bensimon, A., Fillies, M., Domaing, P., Rozanov, Y. M., Leonhardt, H. and Cardoso, M. C. (2016). 4D visualization of replication foci in mammalian cells corresponding to individual replicons. Nat. Commun. 7: 11231. Natale, F., Rapp, A., Yu, W., Maiser, A., Harz, H., Scholl, A., Grulich, S., Anton, T., Hörl, D., Chen, W., Durante, M., Taucher-Scholz, G., Leonhardt, H. and Cardoso, M. C. (2017). Identification of the elementary structural units of the DNA damage response. Nat. Commun. 8: 15760. Heinz, K. S., Casas-Delucchi, C. S., Török, T., Cmarko, D., Rapp, A., Raska, I. and Cardoso, M. C. (2018). Peripheral re-localization of constitutive heterochromatin advances its replication timing and impairs maintenance of silencing marks. Nucleic Acids Res.
45: 6112-6128. Heinz, K. S., Rapp, A., Casas-Delucchi, C. S., Lehmkuhl, A., Romero-Fernández, I., Sánchez, A., Krämer, O. H., Marchal, J. A. and Cardoso, M. C. (2019). DNA replication dynamics of vole genome and its epigenetic regulation. Epigenetics Chromatin In Press.

doi: http://dx.doi.org/10.7124/bc.0009C0

\section{Imaging of cellular DNA damage responses}

\section{Jurek Dobrucki}

Department of Cell Biophysics, Faculty of Biochemistry, Biophysics and Biotechnology, Jagiellonian University, Kraków, Poland

jerzy.dobrucki@uj.edu.pl

Induction of DNA damage elicits a complex set of reactions in a mammalian cell, including signaling, recruitment and accumulation of repair factors at the site of damage, undertaking restoration of the original DNA sequence and chromatin higher order structures, and disengagement of the repair factors after completion of the these processes. The recruited repair proteins form a so called repair focus - an assembly of a large number of factors associated with the DNA lesion or a cluster of lesions. It has emerged recently that the recruited repair factors are highly dynamic and that their spatial positions in some repair foci formed in response to double strand DNA breaks are not random, but convey an ordered internal architecture of these ensembles. Contemporary fluorescence microscopy, in all its various embodiments, including live cell imaging, particle tracking, super-resolution imaging, fluorescence correlation spectroscopy and other methods have opened new avenues to understanding the mechanisms and spatial organization of DNA repair processes. I will 